\title{
Cosmic Ray Air Shower Detection with LOPES
}
A. Haungs ${ }^{a *}$, W.D. Apel ${ }^{\mathrm{a}}$, J.C. Arteaga ${ }^{\mathrm{a} \dagger}$, T. Asch ${ }^{\mathrm{b}}$, A.F. Badea ${ }^{\mathrm{a}}$, L. Bähren ${ }^{\mathrm{c}}$, K. Bekk ${ }^{\mathrm{a}}$, M. Bertaina ${ }^{d}$, P.L. Biermann ${ }^{\text {e }}$,J. Blümer ${ }^{\text {af }}$, H. Bozdog ${ }^{\mathrm{a}}$, I.M. Brancus ${ }^{\mathrm{g}}$, M. Brüggemann ${ }^{\mathrm{h}}$,

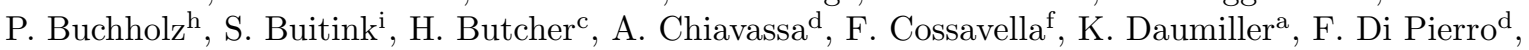 P. Doll ${ }^{\mathrm{a}}$, R. Engel ${ }^{\mathrm{a}}$, H. Falcke ${ }^{\text {cei }}$, H. Gemmeke ${ }^{\mathrm{b}}$, P.L. Ghia ${ }^{\mathrm{j}}$, R. Glasstetter ${ }^{\mathrm{k}}$, C. Grupen $^{\mathrm{h}}$, A. Hakenjos ${ }^{\mathrm{f}}$, D. Heck ${ }^{\mathrm{a}}$, J.R. Hörandel ${ }^{\mathrm{f}}$, A. Horneffer ${ }^{\mathrm{i}}$, T. Huege ${ }^{\mathrm{a}}$, P.G. Isar ${ }^{\S}$, K.-H. Kampert ${ }^{\mathrm{k}}$, Y. Kolotaev ${ }^{\mathrm{h}}$, O. Krömer ${ }^{b}$, J. Kuijpers ${ }^{\mathrm{i}}$, S. Lafebre ${ }^{\mathrm{i}}$, P. Luczak ${ }^{1}$, H.J. Mathes ${ }^{\mathrm{a}}$, H.J. Mayer ${ }^{\mathrm{a}}$, C. Meurer ${ }^{\mathrm{a}}$, J. Milke ${ }^{\mathrm{a}}$, B. Mitrica ${ }^{g}$, C. Morello ${ }^{\mathrm{j}}$, G. Navarra ${ }^{\mathrm{d}}$, S. Nehls ${ }^{\mathrm{a}}$, A. Nigl $^{\mathrm{i}}$, J. Oehlschläger ${ }^{\mathrm{a}}$, S. Ostapchenko ${ }^{\mathrm{q}}$, S. Over ${ }^{\mathrm{h}}$, M. Petcu ${ }^{\mathrm{g}}$, J. Petrovic ${ }^{\mathrm{i}}$, T. Pierog ${ }^{\mathrm{a}}$, S. Plewnia ${ }^{\mathrm{a}}$, J. Rautenberg ${ }^{\mathrm{k}}$, H. Rebel ${ }^{\mathrm{a}}$, M. Roth ${ }^{\mathrm{a}}$,

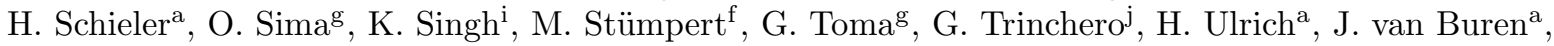 W. Walkowiak ${ }^{\mathrm{h}}$, A. Weindl ${ }^{\mathrm{a}}$, J. Wochele ${ }^{\mathrm{a}}$, J. Zabierowski ${ }^{\mathrm{l}}$, J.A. Zensus ${ }^{\mathrm{e}}$, D. Zimmermann ${ }^{\mathrm{h}}$

\section{LOPES Collaboration}

${ }^{a}$ Institut für Kernphysik, Forschungszentrum Karlsruhe, D-76021 Karlsruhe, Germany

${ }^{b}$ Inst. Prozessdatenverarb. und Elektronik, Forschungszentrum Karlsruhe, D-76021 Karlsruhe, Germany cASTRON, 7990 AA Dwingeloo, The Netherlands

dDipartimento di Fisica Generale dell'Università,10125 Torino, Italy

eMax-Planck-Institut für Radioastronomie, 53010 Bonn, Germany

${ }^{\mathrm{f}}$ Institut für Experimentelle Kernphysik, Universität Karlsruhe, D-76021 Karlsruhe, Germany

${ }^{g}$ National Institute of Physics and Nuclear Engineering, P.O. Box Mg-6, RO-7690 Bucharest, Romania

${ }^{\text {h}}$ Fachbereich Physik, Universität Siegen, 57068 Siegen, Germany

${ }^{i}$ Dept. of Astrophysics, Radboud University Nijmegen, 6525 ED Nijmegen, The Netherlands

${ }^{j}$ Istituto di Fisica dello Spazio Interplanetario, INAF, 10133 Torino, Italy

${ }^{k}$ Fachbereich Physik, Universität Wuppertal, 42097 Wuppertal, Germany

${ }^{\text {l} S o l t a n ~ I n s t i t u t e ~ f o r ~ N u c l e a r ~ S t u d i e s, ~ P L-90950 ~ L o d z, ~ P o l a n d ~}$

LOPES is set up at the location of the KASCADE-Grande extensive air shower experiment in Karlsruhe, Germany and aims to investigate radio pulses from extensive air showers experimentally and theoretically. Data taken during half a year of operation of 10 LOPES antennas (LOPES-10), triggered by EAS observed with KASCADE-Grande have been analysed. We report about the results of correlations with shower parameters present in the radio signals measured by LOPES-10. The extended setup LOPES-30 consists of 30 antennas which have an absolute calibration and the data of which will be compared with expectations from detailed Monte-Carlo simulations. In addition, LOPES operates antennas of a different type (LOPES ${ }^{\text {STAR }}$ ) which are optimized for an application at the Pierre Auger Observatory.

\section{Introduction}

The traditional method to study extensive air showers (EAS) is to measure the secondary particles with sufficiently large particle detector arrays. In general these measurements provide only

\footnotetext{
*corresponding author, e-mail: haungs@ik.fzk.de

${ }^{\dagger}$ permanent address: CINVESTAV, Mexico D.F., Mexico

$\ddagger$ on leave of absence from $g$

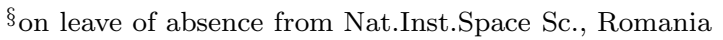

ฯ on leave of absence from Moscow State University, Russia
}

information on the actual status of the air shower cascade on the particular observation level. This hampers the determination of the properties of the EAS inducing primary as compared to methods like the observation of Cherenkov and fluorescence light [1]. In order to reduce the statistical and systematic uncertainties of the detection and reconstruction of EAS, especially with respect to the detection of cosmic particles of highest energies, there is a current methodical discussion on 
new detection techniques. Due to technical restrictions in past times the radio emission accompanying cosmic ray air showers was a somewhat neglected EAS feature. However, the study of this EAS component has experienced a revival by recent activities.

This contribution sketches briefly the activities of the LOPES project [2]. The main emphasis is put on detailed investigations of the correlations of the registered radio signals with the propoerties of the primary cosmic particles by measuring in coincidence with the EAS registration of the running EAS experiment KASCADEGrande [3]. KASCADE-Grande is an extension of the multi-detector setup KASCADE (KArlsruhe Shower Core and Array DEtector) built in Germany [4], measuring air showers in the primary energy range of $100 \mathrm{TeV}$ to $1 \mathrm{EeV}$ with high precision due to a dense grid of detector stations. Hence, LOPES, which is designed as digital radio interferometer using large bandwidths and fast data processing profits from the reconstructed air shower observables of KASCADEGrande. In the frame of LOPES, also theoretical studies of the radio emission are performed in the scheme of the so-called coherent geosynchrotron radiation. Here, electron-positron pairs generated in the shower development gyrate in the Earth's magnetic field and emit radio pulses by synchrotron emission [5]. During the shower development the electrons and positrons are concentrated in a thin shower disk $(<2 \mathrm{~m})$, which is smaller than one wavelength (at $100 \mathrm{MHz}$ ) of the emitted radio wave. This situation provides the coherent emission of the radio signal.

\section{Simulations of the radio emission}

Detailed analytical [5] and Monte-Carlo simulations [6] lead to expectations of relevant radio emission at frequencies of $10 \mathrm{MHz}$ to $500 \mathrm{MHz}$ with a coherent emission at low frequencies up to $100 \mathrm{MHz}$. For showers above a threshold energy of $\approx 5 \cdot 10^{16} \mathrm{eV}$ one expects a short, but coherent radio pulse of $10 \mathrm{~ns}$ to $100 \mathrm{~ns}$ with an electric field strength significantly above the galactic noise and proportionally increasing with the primary energy of the cosmic particle initializing the

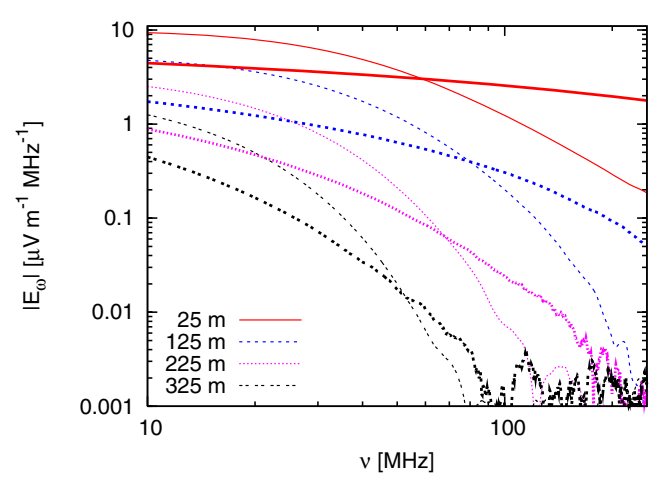

Figure 1. Overall change in the radio signal frequency spectra from a fully parametrised (thin lines) to a fully histogrammed (thick lines) air shower simulation at various distances to the north from the shower center (vertical EAS, $\left.10^{17} \mathrm{eV}\right)$ [7].

air shower.

The above mentioned Monte Carlo simulations were based on an air shower model using analytical parametrisations to describe the spatial, temporal, energy and angular distributions of the air shower particles. The new Monte Carlo code, presented first in [7], replaces these analytical parametrisations with multidimensional histograms generated for each individual shower by the air shower simulation code CORSIKA [8], allowing a much more precise calculation of the radio signal. In particular, this new simulation strategy handles now realistic, histogrammed particle distributions and eliminates a remaining free parameter (track length) that was present in the earlier simulations. Changes between the old and new code are shown exemplarily on the emitted spectra of the air shower in Fig. 1. In addition, the new code can now be used to explain the shape of the emitted radio pulses, e.g. the contribution of different phases in the shower development to the pulse as shown in Fig. 2.

\section{General layout and data processing}

The basic idea of the LOPES (= LOFAR prototype station) project is to build an array of relatively simple, quasi-omnidirectional dipole antennas, the received waves are digitized and sent 


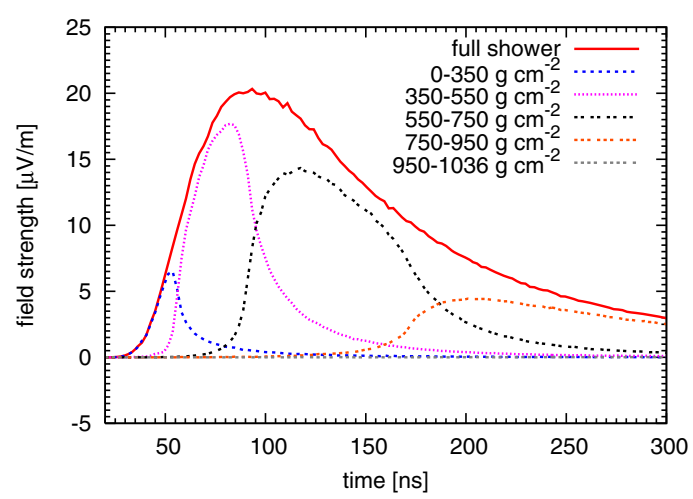

Figure 2. Contribution of different shower evolution stages to the simulated radio pulse at 525 $m$ north from the shower center (vertical EAS, $\left.10^{17} \mathrm{eV}\right)[7]$.

to a central computer. This combines the advantages of low-gain antennas, such as the large field of view, with those of high-gain antennas, like the high sensitivity and good background suppression. With LOPES it is possible to store the received data stream for a certain period of time, i.e. at a detection of a transient phenomenom like an air shower retrospectively a beam in the desired direction can be formed. The air shower experiment KASCADE-Grande provides a trigger of high-energy events and additionally with its direction reconstruction a starting point for the radio data analyses and the beam forming.

In the current status LOPES operates 30 short dipole radio antennas (LOPES-30) having now an absolute calibration. Data of the first 10 antennas forming LOPES-10 have so far been analysed. All LOPES-30 antennas are deployed in east-west direction, measuring the east-west polarisation, only. In a new measuring campaign, also dualpolarized LOPES antennas will be used. In addition, LOPES runs a field of logarithmic-dipoleantennas (LPDA) which are optimized for an application at the Pierre-Auger-Observatory, and for developing a selftrigger system (LOPES ${ }^{\mathrm{STAR}}$ ). The layout is depicted in Fig. 3. All the antennas operate in the frequency range of $40-80 \mathrm{MHz}$. The read out window for each LOPES-30 antenna is $0.8 \mathrm{~ms}$ wide, centered around the trigger received from the KASCADE array. The sam-

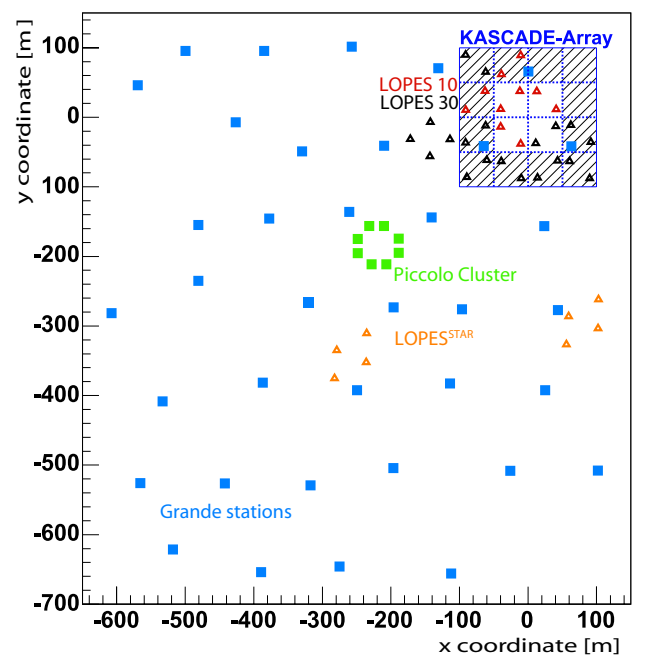

Figure 3. Sketch of the KASCADE-Grande LOPES experiment: The KASCADE array, the distribution of the 37 stations of the Grande array, and the small Piccolo cluster for fast trigger purposes are shown. The location of the 30 LOPES radio antennas is also displayed as well as the LOPES ${ }^{\text {STAR }}$ antennas.

pling rate is $80 \mathrm{MHz}$. The geometry of the antenna and the aluminum ground screen give the highest sensitivity to the zenith and half sensitivity to zenith angles of $43^{\circ}-65^{\circ}$, dependent on the azimuth angle. LOPES-30 data are read out if KASCADE triggers by a high multiplicity of fired stations, corresponding to primary energies above $\approx 10^{16} \mathrm{eV}$. Such showers are detected at a rate of $\approx 2-3$ per minute.

The LOPES data processing includes several steps [9]. First, the relative instrumental delays are corrected using a known TV transmitter visible in the data. Next, the digital filtering, gain corrections and corrections of the trigger delays based on the known shower direction (from KASCADE) are applied and noisy antennas are flagged. Then a time shift of the data is done and the combination of the data is performed calculating the resulting beam from all antennas. This geometrical time shift (in addition to the instrumental delay corrections) of the data, is the time difference of the pulse coming from the given direction to reach the position of the correspond- 
ing antenna compared to the reference position. This shift is done by multiplying a phase gradient in the frequency domain before transforming the data back to the time domain. The data is also corrected for the azimuth and zenith dependence of the antenna gain. A crucial element of the detection method is the digital beam forming which allows to place a narrow antenna beam in the direction of the cosmic ray event. To form the beam from the time shifted data, the data from each pair of antennas is multiplied time-bin by timebin, the resulting values are averaged, and then the square root is taken while preserving the sign. We call this the cross-correlation beam or CCbeam. Finally, there is a quantification of the radio shower parameters: Although the shape of the resulting pulse (CC-beam) is not really Gaussian, fitting a Gaussian to the smoothed data (by block averaging over 3 samples) gives a robust value for the peak strength, which is defined as the height of this Gaussian. The error of the fit results gives also a first estimate of the uncertainty of this parameter. The finally obtained value $\epsilon_{\nu}$, which is the measured amplitude divided by the effective bandwidth, is compared with further shower observables from KASCADE-Grande, e.g. the angle of the shower axis with respect to the geomagnetic field, the electron or muon content of the shower, the estimated primary energy, etc.

\section{Results of LOPES-10}

The LOPES-10 data set corresponds to a measuring period of seven months and has been subject of various analyses addressing different scientific aspects. With a sample of high quality events the proof of principle for detection of air showers in the radio frequency range has been achieved [2].

From the triggered events falling inside the area of the original $200 \times 200 \mathrm{~m}^{2}$ large KASCADE array more than 220 events with a clear radio signal could be detected. The analysis of these events concentrates on the correlations of the radio signal with all shower parameters, in particular with the arrival direction and with the shower size, as well as the primary energy of the shower. As example, Fig 4 depicts the dependence of the recon-

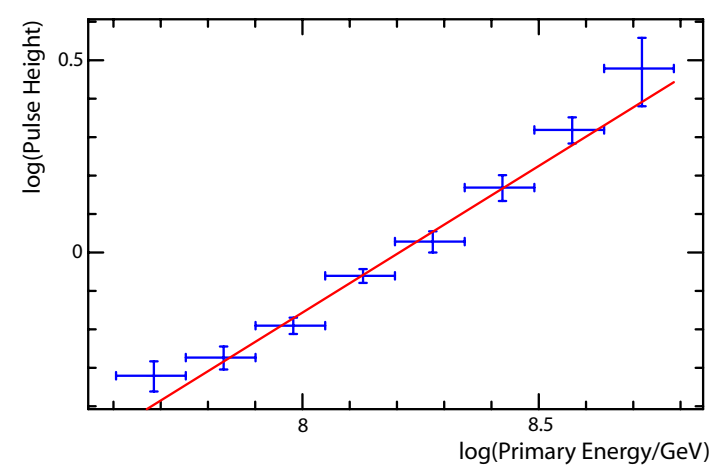

Figure 4. Radio pulse height of the LOPES10 detected events (with shower core inside the KASCADE array and corrected for the geomagnetic angle) plotted versus the primary particle energy as reconstructed by KASCADE [9].

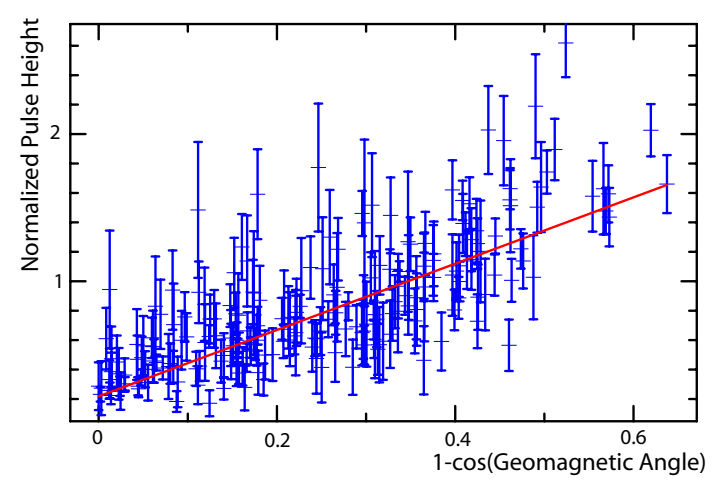

Figure 5. Radio pulse height (corrected for muon number and distance to the shower axis) versus the cosine of the angle to the geomagnetic field. The error bars are the statistical errors [9].

structed averaged radio pulse height on the primary energy of the cosmic particles. The shown correlation supports the expectation that the field strength increases by a power-law with an index close to one with the primary energy, i.e. that the received power of the radio signal increases quadratically with the primary energy of the cosmic rays.

Fig. 5 shows the correlation between the normalized reconstructed pulse height of the events with the geomagnetic angle. Normalized here means, that the detected pulse height is corrected for the dependence on the muon number, i.e. to a 
large extent, the primary energy, and distance to the shower axis. The clear correlation found suggests a geomagnetic origin for the emission mechanism.

Besides the analyses of events with the core inside the antenna setup, KASCADE-Grande gives the possibility to search for distant events. For each (large) shower triggering KASCADE, the information from the extension of KASCADE, i.e. from the Grande array, is available. From that information the shower can be reconstructed even if the core is outside the original KASCADE area, and a radio signal can be searched for events which have distances up to $800 \mathrm{~m}$ from the center of the antenna setup. LOPES-10 detects clear EAS radio events at more than $500 \mathrm{~m}$ distance from the shower axis for primary energies below $10^{18} \mathrm{eV}$. That itself is a remarkable result, but in addition, an important issue is the functional form of the dependence of the radio field strength with distance to the shower axis. After linear scaling of the pulse amplitude with the primary energy estimated by KASCADE-Grande a clear correlation with the mean distance of the shower axis to the antennas is found (Fig. 6). This correlation can be described by an exponential function with a scaling radius in the order of a few hundred meters [10].

Further interesting features are investigated with a sample of very inclined showers [11] and with a sample of events measured during thunderstorms [12]. The former sample is of special interest for a large scale application of this detection technique, as due to the low attenuation in the atmosphere also very inclined showers should be detectable with high efficiency. With LOPES one could show that events above $70^{\circ}$ zenith angle still emit a detectable radio signal. The measurements during thunderstorms are of interest to investigate the role of the atmospheric electric field in the emission process.

\section{Extension to LOPES-30}

LOPES-30 (see Fig.3) has now a maximum baseline of approximately $260 \mathrm{~m}$ by the addition of 20 new antennas. The antennas have an absolute calibration, and the array provides a larger

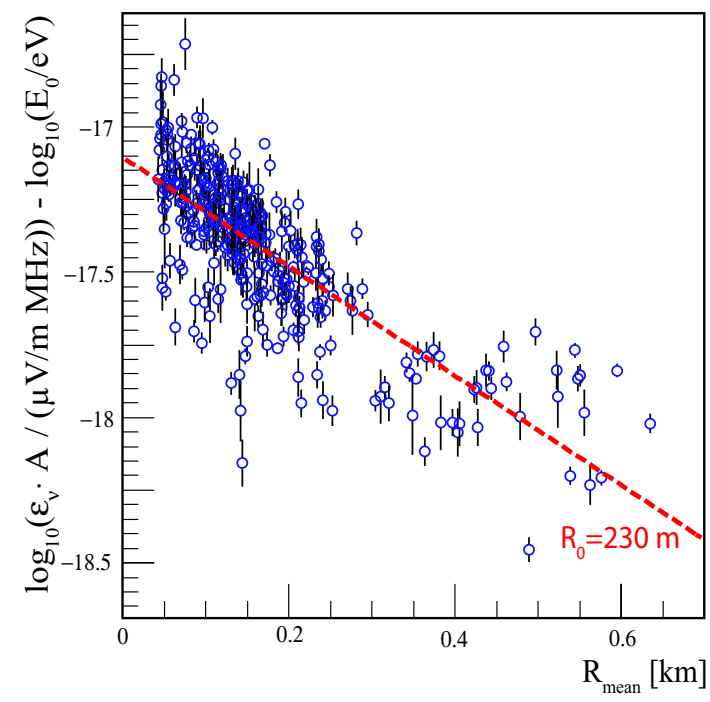

Figure 6. Correlation (sample of distant events) of the pulse height corrected for primary energy with the mean distance of the shower axis to the radio antenna system. The line shows the result of a fit with an exponential function [10].

sampling area to the radio signal of a single event compared with the original LOPES-10 set-up. This provides the possibility for a more detailed investigation of the radio signal on a single air shower basis, in particular of its lateral extension.

Each single antenna is absolute calibrated using a commercial reference antenna [13]. The calibration leads to frequency-dependent amplification factors (Fig. 7), representing the amplification of the electronics. These correction factors are applied to the measured signal strengths resulting in the true electric field strength which can be compared to the values predicted by simulations. By these calibration procedures variations between different antennas are corrected for. Remaining differences (due to variations in the individual antenna gains) are estimated to be less than $25 \%$, which provides also a first estimate of a systematic uncertainty in the measurements.

In addition, during the LOPES-30 measurements, we put emphasis on monitoring environmental conditions by measuring the static electric field and by recording parameters of nearby weather stations. Atmospheric conditions, in par- 


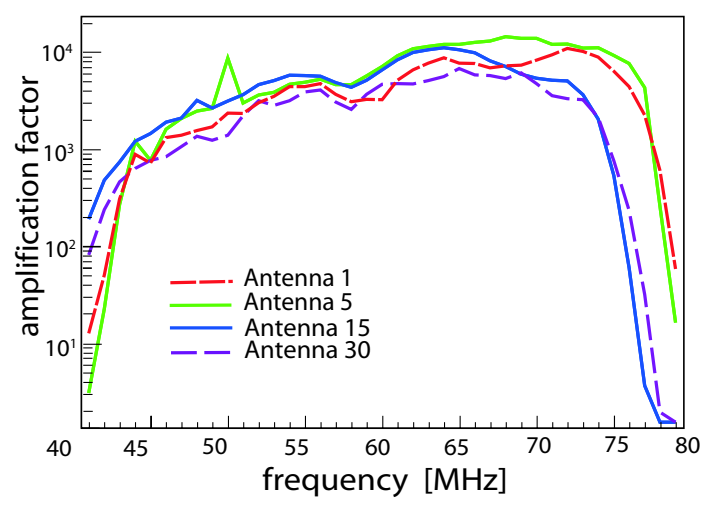

Figure 7. Example for the frequency dependent amplification factors, including full influence of both, individual antenna electronics and environmental conditions. The peak at $50 \mathrm{MHz}$ of antenna 5 is a faulty measurement due to amateur radio activities.

ticular E-field variations during thunderstorms, might influence the radio emission during the shower development, and the measurement of the radio pulses [12]. By monitoring the environmental conditions, and comparing them with the antenna noise level as well as with the detected air shower radio signals, correlations will be investigated and corrected for.

\section{Summary}

LOPES is runs and continuously takes data in an event-by-event coincidence with the air shower experiment KASCADE-Grande. The first results are very promising with respect to the proof of detection of radio flashes from cosmic rays.

With LOPES-10 events with primary energies even below $10^{17} \mathrm{eV}$ and events at more than $500 \mathrm{~m}$ distance from the shower axis were detected in the radio domain, which is remarkable considering the noisy environment at the experimental site. In addition, the clear correlation of the measured radio pulses with the geomagnetic angle suggests a geomagnetic origin for the emission mechanism. Finally, the found quadratic dependence of the radio power on the primary energy will make radio detection to a cost effective method for measuring air showers of the highest energy cosmic rays.
With LOPES-30 we will be able to follow the main goal of the LOPES project: The determination of the correlation of the radio emission in extensive air showers with the primary particle properties.

Last, but not least, there are also impressing improvements in the simulation of the radio emission in the frame of the geosynchreotron mechanism, which will be the basis to interpret and to understand the measured radio signals.

Acknowledgments: LOPES was supported by the German BMBF and is part of the research programme of the Stichting voor FOM supported by NWO. KASCADE-Grande is supported by the MIUR of Italy, the Polish Ministry of Science and Higher Education and the Romanian National Academy for Science, Research and Technology.

\section{REFERENCES}

1. A. Haungs, H. Rebel, M. Roth, Rep. Prog. Phys. 66 (2003) 1145.

2. H. Falcke et al. - LOPES Collaboration, Nature 435 (2005) 313.

3. G. Navarra et al. - KASCADE-Grande Coll., Nucl. Instr. Meth. A 518 (2004) 207.

4. T. Antoni et al. - KASCADE Collaboration, Nucl. Instr. Meth. A 513 (2003) 429.

5. T. Huege and H. Falcke, Astronomy \& Astrophysics 412 (2003) 19.

6. T. Huege and H. Falcke, Astropart. Phys. 24 (2005) 116.

7. T. Huege, R. Engel, and R. Ulrich, Proc. of the ARENA 2006 workshop, Newcastle, UK, preprint astroph-0609809

8. D. Heck et al., Report FZKA 6019, Forschungszentrum Karlsruhe (1998).

9. A. Horneffer, Rheinische Friedrich-WihelmsUniv. Bonn, Germany, 2006, http://nbnresolving.de/urn:nbn:de:hbz:5N-07816

10. W.D. Apel et al. - LOPES Collaboration, Astropart. Phys. 26 (2006) 332.

11. J. Petrovic et al. - LOPES Collaboration, Astronomy \& Astrophysics 2006, in press.

12. S. Buitink et al. - LOPES Collaboration, Astronomy \& Astrophysics 2006, submitted.

13. S. Nehls et al. - LOPES Collaboration, Int. Journ. Mod. Phys. A 21 Suppl. (2006) 187. 